Structural transitions in crystalline foams

This article has been downloaded from IOPscience. Please scroll down to see the full text article. 1998 Europhys. Lett. 41571

(http://iopscience.iop.org/0295-5075/41/5/571)

View the table of contents for this issue, or go to the journal homepage for more

Download details:

IP Address: 129.20.78.138

The article was downloaded on 14/03/2011 at 14:46

Please note that terms and conditions apply. 
Europhys. Lett., 41 (5), pp. 571-576 (1998)

\title{
Structural transitions in crystalline foams
}

\author{
P. Boltenhagen and N. Pittet $\left(^{*}\right)$ \\ Laboratoire d'Ultrasons et de Dynamique des Fluides Complexes, URA 851 \\ Université Louis Pasteur - 4 rue Blaise Pascal, F-67070 Strasbourg Cedex, France
}

(received 2 April 1997; accepted in final form 15 January 1998)

PACS. 82.70Rr - Aerosols and foams.

PACS. $68.90+\mathrm{g}-$ Other topics in structure, and nonelectronic properties of surfaces and interfaces; thin films and whiskers.

PACS. 61.72-y - Defects and impurities in crystals; microstructure.

\begin{abstract}
In this letter, the structural stability of foam is investigated experimentally. We use crystalline foams (equi-sized gas bubbles confined in a cylindrical glass tube) under conditions of a steady-state drainage where soap solution is added onto the top of the foam at a constant rate. By varying this flow rate, we observe: 1) Reproducible structural transitions that are induced when drainage exceeds a critical liquid flow rate. The transition leads to a new ordered foam stable under the flow. A structural diagram is defined, showing the boundary between the two structures for the whole range of existence of the initial structure. 2) A deviation from the classical Poiseuille behavior of the draining liquid, exhibited at low flow rates, is observed. 3) We propose a simple model for the flow into the foam, explaining both the deviation from the Poiseuille behavior and the stability boundary shown in the structural diagram.
\end{abstract}

Introduction. - Pouring a pint of beer is one of the simplest experiments that can be performed to examine the fascinating physics of foam. It is observed that the height of the foam reduces with time, due to coarsening and coalescence of the bubbles [1], [2]. Also the foam is wetter at the bottom than at the top which is due to a phenomenon called the free drainage: The liquid which is contained in the foam flows gravity-driven from the top to the bottom of the foam until it reaches an equilibrium. This phenomenon has been the subject of various studies [3], [4] and was mainly treated phenomenologically.

Recently the drainage at constant flow as studied by Weaire et al. [5] has led to and confirmed analytical theories [6], [7]. In those experiments an amorphous foam is formed inside a cylindrical tube and wetted from the top with a soap solution at a given flow rate. It has been demonstrated that at low flow rate, the flow is meanly controlled by the Plateau borders, channels of liquid at the intersection of three soap films, and thus that the flow is essentially Poiseuille like.

What happens at intermediate and high flow rates? Has the real structure an influence on the drainage or is the Plateau border approximation sufficient to understand drainage? What kind of structural reorganization occurs when a foam is increasingly wetted until the complete loss of rigidity? To answer these questions, we found of great interest to use crystalline foams [8], [9]. They allow to link a direct local observation of the structure and its changes with flow measurements. Also the crystalline aspect amplifies the physical effects of the drainage.

In this letter, we focus our attention on the reorganizations induced by the flow and the departure from the Poiseuille flow regime at higher flow rates. In order to do so, we create

$\left(^{*}\right)$ Present address: GMCM, Université de Rennes I, 35042 Rennes Cedex, France.

(C) EDP Sciences 
ordered structures inside a cylindrical tube and examine their evolution with the flow. We show that: 1) Above a critical flow rate, structural transitions are induced by the flow. The transition leads to a new ordered structure. The mechanism of the transition is explained by the creation and diffusion of a single defect. 2) A structural phase diagram can be built, showing the boundary between the two observed structures. 3) The liquid contained inside the foam depends on the flow rate and exhibits a departure from the standard Poiseuille regime known at low flow rates. A simple model is proposed to explain both the phase boundary and the departure from the Poiseuille regime in the flow curves.

Experimental facts. - During the course of one experiment, ordered foams are created by using the experimental set-up first described by Weaire et al. [5]: Equi-sized air bubbles made from a soap solution are introduced into a cylindrical transparent glass tube. The packing of these identical objects into a geometrically constrained space leads to a crystalline arrangement. It has been shown [8], [9] that in a range of bubble's radii $\left(r_{\mathrm{b}}\right)$ keeping the cylinder's radius $(R)$ constant, one is able to produce topologically equivalent structures. Each structure is labelled by 3 integers $k, l, m$ (for more details see [10], [9]) as usually done in crystallography and by a dimensionless number $\lambda_{0}=R / r_{\mathrm{b}}$.

In practice, bubbles are created by blowing air through a thin nozzle. By controlling the air flow with a manometer, we are able to produce bubbles with a constant radius varying from 0.1 to $10 \mathrm{~mm}$. The initial height $\left(h_{0}\right)$ of the foam is chosen to be $25 \mathrm{~cm}$ over the level of the soap solution. This height corresponds to the height of the dry foam when there is no flow. In this state, the foam is essentially all gas. The structure is then wetted by its top using the same soap solution. The rate of drainage as controlled by a precise tunable pump ranges from $0 \mathrm{~g} / \mathrm{min}$ to $65 \mathrm{~g} / \mathrm{min}$ with a $0.2 \mathrm{~g} / \mathrm{min}$ accuracy.

By varying the flow rate $Q$, one observes first a dilation of the structure which extends below the level of the soap solution: The length of the immersed part $(\Delta h)$ of the foam is measured as a function of $Q$. The top of the foam shows no displacement under flow. Using Archimedes law, the volume fraction of liquid $\phi_{1}=\Delta h /\left(\Delta h+h_{0}\right)$ is calculated as a function of $Q$, and curves $\phi_{1}(Q)$ are obtained. It has been shown [11] that this way of measuring $\phi_{1}(Q)$ is sufficiently accurate and direct to calculate the volume fraction of liquid.

Above a critical flow rate, structural transitions are observed. The initially ordered structure is transformed into another ordered structure.

Structural transitions. - We have systematically investigated the structural transitions induced by forced drainage on the structure shown in fig. 1. This is a flat symmetric structure labelled 4, 2, 2 known [8] to exist in a dry state in the range $1.54<\lambda_{0}<1.98$. This arrangement is a packing of truncated minimal Kelvin tetrakaidecahedra with a hexagonal pattern at the surface of the tube and square faces in the middle of the tube. Once a 4, 2, 2 structure is formed inside the tube, we wet it from its top and gradually increase the flow rate, while waiting until the structure is stabilized. Once the structure is in equilibrium, the same procedure is repeated. Above a critical flow rate, a structural transition is observed: The 4, 2, 2 structure is transformed into a $3,2,1$ one (see fig. 1).

In the whole range of existence of the dry 4,2, 2 structure the same kind of transition is observed. Moreover the critical flow rate $\left(Q_{\mathrm{c}}\right)$ is found to be a function of the dimensionless parameter $\lambda_{0}$. We shall present later a complete study of this dependence in due course.

The mechanism of such a transition is a sequence of topological transformations made possible by the increase of $Q$. Figure 2 shows a sketch of the mechanism responsible for the transition. The first step is a loss of one edge of the inner square face (T1 process) followed by the disappearance of a triangular face (T2 process). The first local rearrangement ( $\mathrm{T} 1$ and T2) is always observed at the top of the tube. This is due to the small variation in the flow 


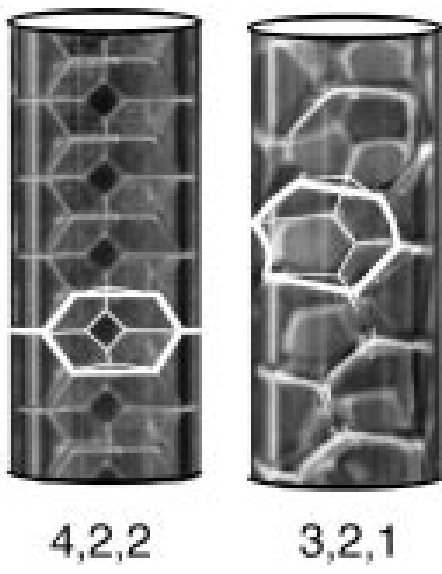

Fig. 1

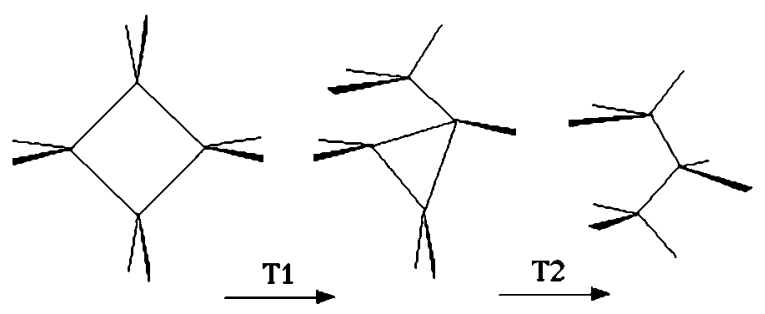

Fig. 2

Fig. 1. - Photographs of a 4, 2, 2 (left) and a 3, 2, 1 structure (right). Thick white lines show the structure at the surface of the tube made of hexagons, then thin lines show the internal structure (Plateau borders) of one bubble. The 4, 2, 2 structure is a packing of truncated Kelvin polyhedra. The diameter of the tube is about $1 \mathrm{~cm}$. By changing the rate of drainage, the structural transition $4,2,2-3,2,1$ is observed.

Fig. 2. - Sketch of the topological transformation occurring during the structural transition. The first step is the loss of one edge of the internal square face (T1 process), followed by the disappearance of the triangular face (T2 process). This sequence of local switches moves from the top of the structure to the bottom. At the end of the process the 4, 2, 2 structure is changed into the $3,2,1$ one.

rate that makes the first switch possible. The destabilization occurs first at the top of the tube and initiates the transition from the top to the bottom of the tube. The succession of these local rearrangements defines a boundary between the 3,2,1 and the 4,2, 2 structure which moves from the top of the tube to its bottom.

At the end of the process, all the 4,2, 2 structure is transformed into a 3,2, 1 structure stable under drainage. When the flow rate is further stopped before the 3, 2, 1 structure occupies the entire tube, we have observed that the $3,2,1$ structure is no longer stable and transforms back to the 4, 2, 2 structure, but no systematic study has been done. This is the first time that such reproducible transitions are observed, explained and quantified. We have not observed stable coexistence of both structures under drainage. When the liquid fraction is increased further, we observe a transition from the $3,2,1$ structure to the $3,3,0$ and for higher liquid fraction, we observe the rigidity loss of the structure (long-range motions of bubbles are visible).

As already said, the driving parameter of this transition is the flow rate, $Q$. When the liquid drains at low flow rate corresponding to low liquid fraction, Verbist et al. [12] have shown that the liquid flow is mainly located in the Plateau borders and thus we can assume very few changes in the morphology of the foam. At higher flow, Plateau borders become thicker, the polyhedral cells more rounded and thus the fourfold junctions larger and closer to each other. At even higher flow rate, geometrical changes are expected when two junctions are close enough to allow the first local topological transformation: This will define our critical flow rate. We have systematically investigated the dependence on $Q_{\mathrm{c}}$ with $\lambda_{0}$. This has been done by creating dry 4, 2, 2 foams in the whole range of existence of this family of structures. Results are represented on the plot $\left(\lambda_{0}, Q_{\mathrm{c}}\right)$ (see fig. 3). The curve defines the boundary of stability between the 4,2, 2 and the 3, 2, 1 structures: Below the curve, each 4, 2, 2 structure is stable under drainage. 

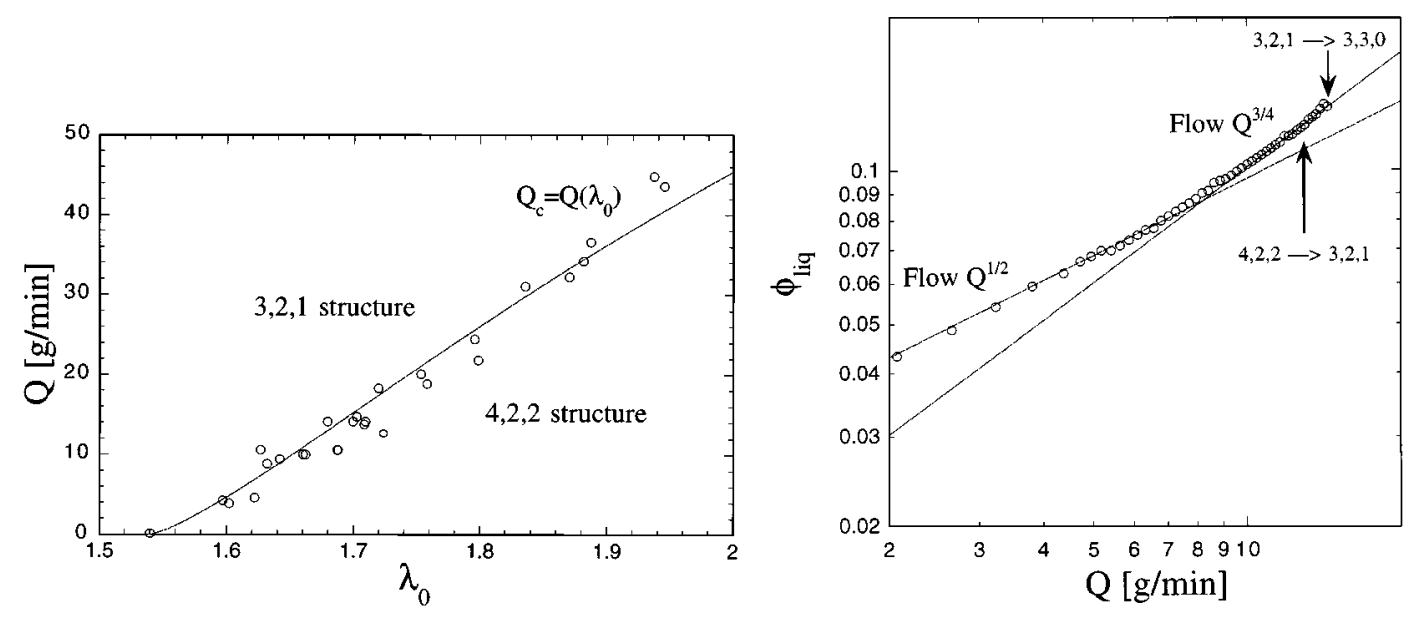

Fig. 3

Fig. 4

Fig. 3. - Structure diagram in the space $\left(\lambda_{0}, Q_{\mathrm{c}}\right)$. The points display the experimental boundary between the 4,2,2 structure and the 3,2,1 structure. The line is the theoretical boundary obtained by fitting eq. (5).

Fig. 4. - Liquid volume fraction $\phi_{1}(Q)$ for the $4,2,2,\left(\lambda_{0}=1.72\right)$ structure as a function of the rate of the drainage $Q$. The two lines show the limiting case for very low and high flow rates derived from eq. (4). The big arrow shows the position of the structural transition $4,2,2-3,2$, 1, the small one the location of the $3,2,1-3,3,0$ transition.

Flow behavior. - It is well established experimentally [5], [13] and theoretically [6], [12], [7], that at low flow rate, the liquid fraction $\phi_{1}(Q)$ behaves by the scaling law $\phi_{1}(Q) \propto Q^{1 / 2}$. This result can be derived as follows: At low flow rate the morphology of the foam is very little perturbed by the liquid. Hence in a good approximation, a simple way to describe the foam is to model the foam structure only by only taking into account the Plateau borders, where the liquid is assumed to flow. The simplest model is $N$-independent vertical parallel elastic pipes in which the flow is Poiseuille-like (i.e. $Q \propto r^{4}$, where $r$ is the radius of the pipes). It follows that the liquid fraction reads

$$
\phi_{1} \propto r^{2} \propto Q^{1 / 2}
$$

We present in fig. 4 a $\phi_{1}(Q)$ curve obtained for one typical 4, 2, 2 structure. We have plotted the liquid fraction as obtained by the method described in the experimental section as a function of the flow rate. We observe that the Poiseuille regime is only valid at low $Q$ : A departure from $Q^{1 / 2}$ is observed at high flow rates. The model of the liquid flowing through the pipes only is then found to be too restrictive to describe the high flow rate behavior. Indeed the liquid can also flow along the faces of the polyhedral cells as well as along the tube wall. However, using simple scaling arguments, those effects would decrease the exponent to $1 / 4$ at high flow rate instead of increasing it. The other alternative for the flowing liquid is to be also located into the fourfold junctions. The volume of the junction increases with the liquid content and thus can be a perturbation of the Poiseuille behavior.

A simple model for the flow and the transitions. - Here, we present a very simple way to model the foam, taking into account the fourfold junctions: Following the assumption of Weaire et al. [5], Plateau borders are still modelled as $N$ vertical cylindrical pipes with a radius $r$. The junctions are idealized as spheres with a radius proportional to that of the pipes. These spheres are distributed along the pipes with a constant distance. 
This description gives a periodic foam with a distance between the centers of two adjacent spheres equal to the typical Plateau border length separating two fourfold junctions in a dry foam. The spheres and the pipes behave differently under the liquid flow: In the pipes, the flow is still Poiseuille-like, but the volume of liquid contained in the junctions scales as $Q^{3 / 4}$ (a junction is modelled by a sphere). Taking into account the contribution of both pipes and junctions, we find that the liquid fraction reads

$$
\phi_{\mathrm{l}}(Q)=\frac{V_{\text {pipes }}+V_{\text {junctions }}}{V_{\text {total }}}=\alpha r^{2}+\beta r^{3}=a Q^{1 / 2}+b Q^{3 / 4} .
$$

In fig. $4, Q^{1 / 2}$ and $Q^{3 / 4}$ are plotted in order to show the asymptotic behavior for low and high liquid flow rates before the transition. Our measurements are in the cross-over of the two different regimes and are limited at low flow rates by the accuracy of the pump and at high flow rate by the transition occurring above $Q_{c}$. Equation (2) cannot be applied as a fit in fig. 4 , since the coefficients $a$ and $b$ are dependent on $Q$ in a full description of the cross-over between the low and high flow rates.

For the sake of simplicity and in order to understand the structural phase diagram (fig. 3), we set before the transition that

$$
\phi_{1}\left(Q \rightarrow Q_{\text {transition }}\right) \propto Q^{3 / 4} .
$$

This simple model of a foam and its drainage can be incorporated into a phenomenological model that explains the structural boundary observed and presented in fig. 1. Let us imagine that we create a dry $4,2,2$ structure inside a cylindrical tube. If we gradually decrease the diameter of the tube we will observe first an extension of the foam and some structural transitions at a typical cylinder's radius. This is due to the fact that in this process the value of $\lambda_{0}=R / r_{\mathrm{b}}$ decreases and reaches the value $\lambda_{\mathrm{c}}=1.54$ below which no stable $4,2,2$ structure exists as was found by Pittet et al. [8].

A very close analogy can be done with the drainage experiments that we have performed. When the liquid drains into the structure, the foam is observed to expand below the level of the soap solution and furthermore to transit to another structure. The action of the drainage can be seen as a squeezing of the structure due to the liquid content. We model this effect by making the following assumption: The volume of the liquid contained in the foam decreases the available space of the gas divided in bubbles. We model this effect as a reduction of the tube diameter by putting all the liquid of the foam in a hollow tube surrounding a dry 4 , 2,2 structure. The outer diameter of this hollow tube is equal to the diameter of the tube $D$. The inner one is the diameter available for the structure and is a function of $\phi_{1}(Q)$ (the inside volume of the hollow tube is identified with the liquid volume contained in the foam). Increasing $\phi_{1}(Q)$ leads to a reduction of the available diameter $D_{\text {av }}$ and thus extends the foam vertically. The relation between $\phi_{1}(Q), D$ and $D_{\text {av }}$ is readily obtained from this simple model to give

where $\lambda_{\text {av }}$ is given by

$$
\phi_{\mathrm{l}}(Q)=\frac{\pi\left(D^{2}-D_{\mathrm{av}}^{2}\right)}{\pi D^{2}}=1-\lambda_{\mathrm{av}}^{2}
$$

$$
\lambda_{\mathrm{av}}=\frac{D_{\mathrm{av}}}{D}=\lambda_{0}\left[\left(1-\phi_{\mathrm{l}}(Q)\right]^{1 / 2} .\right.
$$

One can see from eq. (5) that increasing $Q$ will decrease $\lambda_{\mathrm{av}}$. This will define the critical flow rate $Q_{\mathrm{c}}$ which will be a function of $\lambda_{0}: Q_{\mathrm{c}}$ is obtained when $\lambda_{\mathrm{av}}=1.54$. Then, eq. (5) defines the boundary between the 4,2, 2 structure and the 3, 2, 1. Equation (5) can be rewritten in terms of the critical flow rate $\left(Q_{\mathrm{c}}\right)$ as

$$
Q_{\mathrm{c}}=\left[\frac{1}{b}\left(1-\left(1.54 / \lambda_{0}\right)^{2}\right)\right]^{4 / 3} .
$$


The line in fig. 3 is obtained by fitting the experimental points with eq. (6) where $b$ is a free parameter. The general trend of the boundary is well described, nevertheless, the small bending of the experimental points is certainly due to some effects that are difficult to define in the model. Eespecially one would rather take a more accurate description of the fourfold junctions.

Conclusion. - In conclusion, we have demonstrated that reproducible structural transitions are induced in ordered foams by a steady-state drainage. The sequence 4, 2, 2-3,2, 1 is observed and the mechanism of the transition is explained by the increase of the size of the fourfold junction. A structural diagram is built and the stability boundary between the two structures is explained in terms of the reduction of the available diameter of the tube. The next step of in such studies will be the elaboration of a global model to relate these transitions and their dynamical aspects [14], [15].

As a follow-up of this work, it might seem very interesting to study the next transition occurring in this system, namely the transition $3,2,1-3,3,0$, and to build a more complete structural diagram. The mechanism of the complete set of transitions and the stability boundary should give important information about the flow at high rates. Moreover, the loss of rigidity observed in the course of our experiments, with its long-range motion of bubbles is also of interest [14], [15]. We would like to add that the transition between two ordered structures strongly suggests that it may be possible to induce disorder-order transition driven by the drainage. Particularly, if two sizes of bubbles are introduced into the cylindrical tube, it is possible to make a separation of the two families of bubbles. By controlling the flow, the formation of a more complicated ordered structure is possible.

We wish to thank N. Rivier and B. Dubertret for helpful discussions. We would like to thank S. HutzLER for his linguistic contribution to this letter. This research was supported by the EU HCM programme, FOAMPHYS Network, Contract ERBCHRXCT940542. NP was supported by the Swiss OFES, contract OFES94.0066.

\section{REFERENCES}

[1] Bhakta A. and Ruckenstein E., Langmuir, 12 (1996) 3089.

[2] Müller W., Di Meglio J. M., submitted to Europhys. Lett., (1997).

[3] Princen H. M. and Kiss D., Langmuir, 3 (1987) 36.

[4] Bhakta A. and Ruckenstein E., Langmuir, 11 (1995) 1486.

[5] Weaire D., Pittet N., Hutzler S. and Pardal D., Phys. Rev. Lett., 71 (1993) 2670.

[6] Goldfarb I., Kann K. B. and Shreiber I. R., Fluid Dynamics, 23 (official English translation of Transactions of USSR Academy of Sciences, series Mechanics of liquid and Gas) (1988) 244.

[7] Verbist G., Weaire D. and Kraynik A. M., J. Phys. Condens. Matter, 8 (1996) 3715.

[8] Pittet N., Rivier N. and Weaire D., Forma, 10 (1995) 65.

[9] Pittet N., Boltenhagen P., Rivier N. and Weaire D., Europhys. Lett., 35 (1996) 547.

[10] Coxeter H. S. M., Introduction to Geometry, 2nd edition (John Wiley \& Sons, Inc.) 1969.

[11] Pittet N., The Structure of Liquid Foam, MSc Thesis, Trinity College, Dublin, 1993.

[12] Verbist G. and Weaire D., Europhys. Lett., 26 (1994) 631.

[13] Hutzler S., Verbist G., Weaire D. and van der Steen J. A., Europhys. Lett., 31 (1995) 497.

[14] Hutzler S., Weaire D. and Crawford R., submitted (1997).

[15] Hutzler S., Weaire D. and Crawford R., Philos. Mag. B, 75 (1997) 845. 